\title{
LA FORMACIÓN PERMANENTE DEL PROFESOR UNIVERSITARIO EN IBEROAMÉRICA: UNA REVISIÓN CRÍTICA
}

\author{
ONGOING TRAINING OF UNIVERSITY TEACHERS IN \\ IBERO-AMERICA: A CRITICAL REVIEW
}

A FORMAÇÃO PERMANENTE DO PROFESSOR UNIVERSITÁRIO
NA IBERO-AMÉRICA: UMA REVISÃO CRÍTICA

Juan Carlos Padierna Cardona ${ }^{1}$

Margarita Benjumea Pérez²

León Urrego ${ }^{3}$

Resumen

Se presenta una revisión de investigaciones sobre la formación permanente del profesor universitario en diferentes campos de conocimiento con el propósito de identificar contenidos y metodologías desarrolladas por instituciones de educación superior para cualificar el rol profesional de su profesorado. Como ruta metodológica se desarrolló una revisión documental, se analizaron 53 fuentes de revistas científicas obtenidas, en su mayoría, de las bases de datos Dialnet, Ebsco y Scopus en el periodo 1989-2014 usando palabras clave como: formación permanente, desarrollo profesional y profesorado universitario. Se develó que algunos estudios se interesan por desarrollar procesos en pedagogía, didáctica, psicopedagogía, currículo, gestión curricular e investigación, en tanto otros se centran en identificar acciones de los profesores para atender sus intereses de autoformación. Asimismo, se identificaron posibles líneas de intervención desde temáticas y condiciones en las cuales las universidades se podrían ocupar de la formación que requieren sus profesores con base en su calidad de novel, experto o tutor.

Palabras clave: educación física; formación permanente; profesor universitario; desarrollo profesoral; currículo

1 Doctor por la Universidad de Antioquia (2017), magíster en Educación por la Universidad de Medellín (1999), especialista en Administración Deportiva (2004) y licenciado en Educación Física (1997) por la Universidad de Antioquia. Profesor de cátedra del Instituto Universitario de Educación Física de la Universidad de Antioquia. Código Orcid: 0000-0001-8409-9914. Correo electrónico: juan.padierna@ udea.edu.co.

2 Doctora en Educación (2012), magíster en Motricidad y Desarrollo Humano (2009), especialista en Administración Deportiva (2000) y licenciada en Educación Física (1986) por la Universidad de Antioquia. Profesora titular del Instituto Universitario de Educación Física de la Universidad de Antioquia. Código Orcid: 0000-0002-6835-4273. Correo electrónico: margarita.benjumea@udea.edu.co.

3 Doctor por la Universidad de Valladolid (2014), licenciado en Educación Física (2003), profesor asistente del Instituto Universitario de Educación Física de la Universidad de Antioquia. Código Orcid: 0000-0002-1652-3418. Correo electrónico: leon.urrego@udea.edu.co. 


\section{Abstract}

We present a review of research on the ongoing training of university professors in different fields of knowledge to identify contents and methodologies developed by higher education institutions to qualify the professional role of their teaching staff. As a methodological route, the authors carried out a documentary review in which they analyzed 53 sources of scientific journals, mostly obtained from the Dialnet, Ebsco and Scopus databases in 1989-2014, using keywords such as ongoing training, professional development and university faculty. The results revealed that some studies focus in developing processes in pedagogy, didactics, psychology and pedagogy, curriculum, curricular management and research, while others revolve around identifying actions of university teachers to address their self-training interests. Likewise, the authors identified possible lines of intervention from the topics and conditions under which universities could take on the training required by their teachers based on their status as novel, expert or tutor.

Keywords: physical education; ongoing training; university teacher; faculty development; curriculum

\section{Resumo}

Uma revisão da pesquisa sobre a formação permanente do professor universitário em diferentes áreas do conhecimento é apresentada com o propósito de identificar conteúdos e metodologias desenvolvidas por instituições de ensino superior para qualificar o papel profissional de seus docentes. Como rota metodológica uma revisão da literatura foi desenvolvida, fontes obtidas de 53 revistas científicas foram analisadas, principalmente de Dialnet, EBSCO e Scopus no período 1989-2014 usando palavras-chave, tais como: educação continuada, desenvolvimento profissional e corpo docente universitário. Foi revelado que alguns estudos estão interessados em desenvolver processos em pedagogia, didática, psicologia educacional, currículo, gestão curricular e pesquisa, enquanto outros se concentram na identificação de ações de professores para atender seus interesses de auto-educação. Da mesma forma, possíveis linhas de intervenção foram identificadas a partir de temas e condições em que as universidades poderiam cuidar da formação exigida pelos seus professores com base na sua qualidade como um novo, especialista, ou tutor.

Palavras chave: educação física; formação permanente; professor universitário; desenvolvimento de professores; currículo

Fecha de recepción: 16 de diciembre de 2016

Fecha de aprobación: 10 de septiembre de 2018

\section{Para citar este artículo}

Padierna, J.C., Benjumea, M. y Urrego, L. (2018). La formación permanente del profesor universitario en Iberoamérica: una revisión crítica. Lúdica Pedagógica, 27, x-xx. 


\section{INTRODUCCIÓN}

Este estudio se centró ${ }^{4}$ en reconocer fundamentos teóricos que circundan la formación permanente del profesor universitario en diferentes campos de conocimiento. Con la pretensión de identificar metodologías y conceptos que permitieran generar aportes para instituciones de educación superior (IES) y, específicamente, para proyectar estos avances en una propuesta diseñada para el campo disciplinar de la educación física. La revisión servirá como punto de partida para un estudio doctoral sobre la formación del profesorado en Educación Física en el contexto de las Universidades Públicas de la Región de Antioquia en Colombia. Esta acción de cualificación en la formación del profesorado, quizás permita develar mejores formas de acompañamiento al profesorado que allí se desempeña, para que se evidencien las diversas acciones que se podrían realizar desde las IES en pro de la cualificación del rol profesional.

La revisión parte de la postura que plantean Gil Pérez, Belendez Vásquez, Martín García y Martínez Torregrosa (1991) quienes resaltan que la formación permanente de profesores debe centrarse en la suma de conocimientos científicos, psicopedagógicos y didácticos para lograr que la actividad del profesor sea abierta, creativa y refleje el conocimiento de la materia por enseñar.

En la misma perspectiva, Ruiz Corbella (1999) resalta que para afrontar la formación de los nuevos ciudadanos se hace necesario aportar al profesorado una formación adecuada en el contexto en que se orienta la educación, reforzando estrategias de trabajo colaborativo en su tarea de contribuir a la construcción de un futuro mejor. Y desde la mirada de Escudero Muñoz (1999) la formación permanente del profesor universitario (FPPU) es un territorio por explorar, que debe dimensionarse desde lo cultural, lo político y lo estratégico de los procesos en las IES para impactar y mejorar la educación y la sociedad.

4 Esta revisión pertenece a la tesis doctoral La formación permanente del profesor universitario de educación física, adscrita a la línea de investigación en Educación, cuerpo y motricidad del Doctorado en Educación de la Universidad de Antioquia. El desarrollo de la tesis contó con el apoyo de una beca de Colciencias para doctorados nacionales (n. ${ }^{\circ}$ 617-2). Fue registrada en la Facultad de Educación en el acta 217 del Comité de Doctorado, con el código de caso 8271 para la defensa de la candidatura el 25 de septiembre del 2015.
En esta perspectiva, algunos factores importantes en la FPPU están en la vía del mejoramiento de la enseñanza desde sus conocimientos, habilidades, actitudes y valores en su saber disciplinar y desde las posibilidades de actitud crítica y reflexiva que se les posibilita en el análisis de su rol profesional y de la formación permanente que reciben. Ello hace necesaria una mirada a las políticas presentes para la FPPU, su funcionamiento, las posibilidades de reflexión e investigación sobre la práctica misma y la identificación de las necesidades del contexto, donde su participación es de vital importancia desde los diagnósticos para asegurar el desarrollo, la implementación, la divulgación e institucionalización de programas de FPPU que se instauren en las IES, determinando rutas de la formación permanente y la construcción de un perfil profesional en escenarios de cooperación y trabajo compartido con los "recién llegados".

Es así como en esta revisión se analizó una selección de investigaciones acerca de la FPPU en diferentes campos de conocimiento, con el fin de conocer problemas y expectativas de IES y las preocupaciones y dinámicas de formación permanente empleadas por los profesores universitarios para mejorar su rol profesional. El propósito es que los datos obtenidos permitan a las IES identificar posibles rutas por implementar en propuestas o programas institucionalizados de FPPU, en aras de cualificar su ejercicio profesional.

\section{MÉTODO}

Este ejercicio siguió las orientaciones de Carrasco y Calderero Hernández (2000) en la revisión de fuentes documentales para identificar los interrogantes por investigar y resolver, precisar el planteamiento del problema, comprender perspectivas históricas y de contexto, identificar métodos y técnicas usuales. En especial, se implementaron las rutas para identificar los artículos científicos en las bases de datos, los procedimientos para escogerlos y se desarrolló la estrategia de recolección y análisis que partió de la lectura y selección con base en las palabras clave, la identificación de la idea central, las ideas de apoyo y las rutas metodológicas y prospectivas de cada uno de los estudios.

La información extractada de los documentos analizados se clasificó para su lectura a partir de las 
palabras clave; tras este filtro, se hizo una lectura general de la documentación seleccionada, con una interpretación reflexiva y analítica de los artículos elegidos. Se empleó una ficha de análisis documental con factores como bibliografía, temáticas centrales del estudio, metodología de investigación empleada, resultados relevantes y nacionalidad de los autores del artículo. Como estrategia de apoyo, se utilizó el gestor de referencias bibliográficas Mendeley.

Con base en este rastreo, se eligieron para la revisión 60 investigaciones publicadas con los siguientes criterios: de exclusión, se descartaron 7 de ellas por no tener relación directa con la temática del estudio (FPPU); de inclusión, entre los 53 estudios seleccionados, 49 son publicaciones en revistas y 4 son libros producto de investigación y que presentan una de las palabras clave en el título, subtítulo o en el núcleo temático. Luego se procedió a la lectura completa, y luego se realizó su ingreso al gestor de referencias analizando los componentes de las publicaciones incluidas (objetivos, métodos, técnicas, instrumentos, principales conclusiones, rutas prospectivas, aportes conceptuales, asignación de categoría de estudio, construcción de citas desde las ideas generales o las ideas de apoyo y, finalmente, la realización de inferencias por parte del analista).

Las investigaciones abordadas para esta revisión develaron dos líneas de atención centrales en y para la FPPU: por un lado, los procesos adelantados por las IES, y por otro, las estrategias solicitadas o empleadas por profesores de diferentes campos de conocimiento para cualificar su rol profesional. Ambas apuntan esencialmente a elementos teóricos, administrativos, prácticos e investigativos (véase la tabla 1).

Tabla 1.

Categorías inductivas de análisis para la FPPU

\begin{tabular}{ll}
\hline $\begin{array}{l}\text { Procesos en y desde las ies } \\
\text { para la formación del profesorado }\end{array}$ & $\begin{array}{l}\text { Estrategias del profesorado } \\
\text { para su cualificación }\end{array}$ \\
\hline Identificación de problemas & Identificación de preocupaciones-necesidades \\
\hline Delimitación de expectativas & Preferencia de dinámicas de formación \\
\hline
\end{tabular}

Fuente: elaboración propia.

\section{RESULTADOS}

Las rutas de FPPU dan cuenta de hallazgos obtenidos frente al interés del estudio que enmarca esta reflexión, con esto se pretende conocer cómo los agentes, sistemas o estructuras encargadas de dicha formación determinan el conocimiento base en dichos programas. Los aspectos más relevantes develados son: las condiciones que influyen en el diseño de estos programas, las políticas que hacen posible su funcionamiento, los procesos académicos para el desarrollo de actividades, las estrategias administrativas para la generación de cobertura y de participación del profesorado; asimismo, se busca identificar las dinámicas o estrategias para la determinación de temáticas pedagógicas o disciplinares desde necesidades, intereses y posibilidades individuales, grupales, institucionales y socioculturales en contexto.
Las investigaciones analizadas permiten comprender una ruta inicial desde perspectivas teóricas, administrativas, prácticas e investigativas que podrían ayudarnos a responder ¿cuáles son los problemas y expectativas de las IEs frente a la FPPU, y las preocupaciones-necesidades y dinámicas de formación permanente para cualificar el rol profesional del profesorado? Desde este interrogante se piensan, tejen y dinamizan las tensiones o vacíos en los procesos de FPPU. Esto resalta la necesidad y pertinencia de su presencia en las agendas de desarrollo en las IEs, en especial cuando se espera tanto de ellas como de los profesores, acciones ajustadas a diferentes necesidades en docencia, investigación, extensión, proyección social, formación ciudadana y responsabilidad social, entre otras demandas, que se entretejen y permean las realidades que enfrentan estos procesos según el contexto. 
Problemas y expectativas de las IES frente a la FPPU

La FPPU es un elemento central del pensamiento pedagógico moderno, según Vaquero (2000), en tanto que este tipo de educación se emplea en la consolidación de procesos institucionales y en la mejora del desempeño de los profesores universitarios a partir de los sistemas educativos de las sociedades. Mayor Ruiz (2003) considera que esta formación se establece a partir de programas o proyectos estructurados por los sistemas universitarios para atender la cualificación de sus profesores, puesto que así se contribuye a consolidar el perfil profesoral que se espera en cada universidad.

García Ruso (1997) señala dos categorías centrales para la FPPU: Problemas y Expectativas, ambas orientadas hacia las IEs. En la primera, ubica instalaciones, políticas, financiación, estructuras administrativas, entre otras; y en la segunda, reconoce las solicitudes que hacen los diferentes actores de las comunidades educativas para el diseño de procesos de desarrollo profesional.

Investigaciones centradas en los problemas

de las universidades frente a la FPPU

Los problemas a los que se ven enfrentados los departamentos o unidades encargadas de la formación permanente en las IES, según las investigaciones consultadas, exigen definir el proceso en el cual se daría este tipo de formación, la estructura organizacional necesaria para tal propósito, la teoría de conocimiento que fundamenta las diversas propuestas, entre otros elementos; todo ello, adecuado a las características de cada universidad y dirigido a atender las necesidades o expectativas frente al desempeño esperado del profesorado.

En este sentido, Imbernon (1989), Pérez Miranda y Gallego Badillo (2006) y Mayor Ruiz (1997) consideran la FPPU desde perspectivas científicas, pedagógicas y didácticas; cuestionan el pensamiento docente espontáneo, pero fomentan el conocimiento científico, el dominio de diferentes metodologías de enseñanza y conocimientos psicopedagógicos. En los trabajos de Zabalza Beraza $(2000,2003)$ se identifican rupturas y conflictos en departamentos que atienden la formación del profesorado universitario; resaltan el fomento y creación de estos bajo ecosistemas de comunicación y apoyo mutuo que miden su impacto y las condiciones para que la investigación y la enseñanza se den.

Para Ferreres Pavía (2001) la importancia está en brindar tiempos para la formación, investigar en equipos, crear líneas de investigación, evitar mezclar la evaluación de desempeño con la formación permanente, fomentar los incentivos, la cooperación, crear políticas de desarrollo profesoral según el área o departamento y fomentar procesos prácticos en el aula, el laboratorio y la empresa.

En la misma perspectiva, Margalef García y Álvarez Méndez (2005) problematizan oportunidades y dificultades para la FPPU; y plantean para menguar estas últimas la creación de los citados departamentos de formación, acogiéndose al uso de las tecnologías de información y comunicación (TIC), la innovación, el trabajo y aprendizaje cooperativo, procurando ser profesores contextualizados, dispuestos, participativos, reconocidos y valorados.

Finalmente, Madrid Izquierdo (2005) y Biscarri Gassio, Filella Guiu y Jové Monclús (2006) analizan la formación del profesor, los responsables administrativos que la financian y los académicos que la planifican, y detectan factores de percepción relacionados con los cambios en la cultura de la docencia universitaria. Por ello, estos investigadores sugieren estimular el desarrollo de competencias cognitivas, metacognitivas, comunicativas, gerenciales, sociales y afectivas para el profesor universitario (véase también Padierna Cardona, 2010) y que cada departamento responsable de la FPPU cuente con infraestructura y financiación para el reconocimiento al desempeño.

Investigaciones centradas en las expectativas de las universidades frente a la FPPU

La identificación de las expectativas de la comunidad universitaria con respecto al desempeño de sus profesores implica al departamento encargado de la FPPU obtener la información de primera fuente (estudiantes, directivos, docentes y pares académicos) mediante diagnósticos para conocer los factores que han de considerar en la planeación de este tipo de formación, divulgar el conocimiento generado acerca de la percepción social sobre el docente y propiciar su formación en competencias genéricas y profesionales de acuerdo con el contexto. 
En tal sentido Belando Montoro (1999), Escudero Muñoz (1999), Imbernon Muñoz (1999), Hadi y Cabrera (1999), Ruiz Corbella (1999) y Cano González y Revuelta Guerrero (1999) consideran que la FPPU se debe plantear desde el perfil profesional esperado en la universidad contemporánea, reconociendo aspectos como uso de TIC, desarrollo de habilidades para la motivación en jóvenes estudiantes, participación, educación para la ciudadanía, entre otras demandas.

Desde otra perspectiva, Rué (2013) sugiere trabajar en la identidad de la profesión desde indicadores reales en salarios, ascensos y mantenimiento del lugar de trabajo. Este autor expone con preocupación la transición del discurso de la docencia, del profesor y del alumno a discursos de institución, normas, acreditación y competitividad.

En los trabajos de Caballero Rodríguez (2013) se propone la creación de departamentos de formación desde las exigencias de los sistemas de evaluación en IES, acordes con características del perfil esperado del profesorado, posibilitar comunidades de aprendizaje, compartir experiencias exitosas y fomentar la autoevaluación y evaluación institucional. Palomero Pescador (2002) considera que la FPPU se debe concebir en clave de impacto científico, pedagógico y social. Y Marín Sánchez y Teruel Melero (2004), resaltan la necesidad de reconocer la percepción social del profesor en su responsabilidad social y su rol en la docencia y la investigación.

La FPPU, según Piña Loyola et al. (2014) debe responder a necesidades, retos de la sociedad actual y uso de recursos técnicos y tecnológicos. Y para Mérida Serrano (2013) el punto de atención debe ser el trabajo con los agentes del proceso de desarrollo profesional, vinculando estudiantes (futuros docentes) y profesores actuales desde perspectivas reflexivas y participativas.

Entre tanto, Yániz Álvarez (2008), Bozu y Canto Herrera (2009), Bozu (2009) y López Rodríguez (2012) destacan la competencia curricular en la sociedad del conocimiento, desarrollando habilidades profesionales y evaluando el conocimiento construido por diversos actores, desde lo pedagógico-científico, la función docente, su motivación e innovación como profesor universitario novel.
Y finalmente, Zabalza Beraza (2013) focaliza su interés en la labor curricular, el liderazgo y la gestión académico-administrativa. Esto permitirá al profesor adquirir competencias generales de lectura, escritura, expresión, relaciones interpersonales, trabajo en equipo, juicio crítico, uso de bases de datos, dominio de una segunda lengua, desarrollo de la ética, la innovación, la creatividad, la empatía y el perfil profesional.

\section{Estrategias del profesorado para su cualificación profesional: preocupaciones/necesidades y dinámicas de formación permanente}

En la perspectiva de Fraile Aranda $(2004,2010)$ se deben atender "las preocupaciones" que expresa el profesorado novel o experto frente o durante su formación. Y Mayor Ruiz (2003) asume "las dinámicas de formación" como las acciones o actividades que emplea el profesor universitario para su formación permanente; estas pueden enfocarse en la oferta que recibe de su institución, de otra o incluso surgen de intereses que en él se suscitan, evidenciando así un proceso de autoformación.

Investigaciones centradas en las preocupaciones del profesorado frente a la FP

Las preocupaciones del profesorado universitario se identifican mediante diagnósticos que permiten reconocer sus necesidades de formación en cuanto a perfil profesional, bienestar laboral, falencias pedagógicas, psicopedagógicas, didácticas, flexibilidad curricular, innovación, uso de TIC, producción y divulgación del conocimiento, conformación de redes y relaciones interpersonales, entre otras tensiones de los profesores en torno a su cualificación.

Para Coharik Chamlian (2003) los factores de buen desempeño e innovación en la enseñanza se obtienen si se estimula a los profesores a identificar, analizar y reflexionar con pares o colegas respecto a sus dificultades. En tal sentido Medina Moya, Jarauta Borrasca y Urquizu Sánchez (2005) proponen crear una matriz de dominios y comprensión de procesos de enseñanza-aprendizaje en contexto, desde factores obstaculizadores como recursos insuficientes e inadecuados, ausencia de apoyos o reconocimientos institucionales a la innovación y ausencia de una cultura académica basada en la colaboración. 
Para Solá Fernández (2004) el uso de TIC es elemento necesario para la gestión del aula y la relación enseñanza-aprendizaje; por eso, sugiere estimular la comunicación en otros idiomas, la formación didáctica en motivación y cooperación, el pensamiento autónomo y la pluralidad metodológica, fusionando además teoría, práctica e investigación. En los estudios de Sánchez y Flores (2013) los profesores universitarios se deben preocupar por los cambios socioeconómicos, culturales, políticos, tecnológicos y científicos de la sociedad globalizada; promover la lectura de contexto, potenciar el desarrollo por competencias y la actualización desde lo científico, lo técnico y el desarrollo pedagógico, mientras se avanza en relaciones de trabajo cooperativo interdisciplinario.

Desde Rodríguez Izquierdo (2003) se fomenta el uso de las metodologías didácticas, el desarrollo de capacidades en el alumnado y el dominio metodológico de la investigación. Finalmente, Concepción Cuétara, Fernández Cruz y González González (2014) proponen diseñar un cuestionario para identificar necesidades de formación en el profesor universitario y contar con las expectativas de directivos, estudiantes y profesores expertos.

Investigaciones centradas en dinámicas de formación del profesorado

Las dinámicas de formación del profesor universitario se originan en la oferta variada de los programas de formación permanente que brindan las universidades, o también, en la que el profesor gestiona de forma particular, autónoma e independiente, dado su interés como docente novel, experto o tutor de dar continuidad a sus necesidades profesionales y al fortalecimiento profesional continuo.

En esta dirección, Giner Cotino y Giner Cotino (1998), Tomé (2000) y García Nieto (2008) plantean que la FPPU se fortalece con propuestas teórico-prácticas para un adecuado asesoramiento o tutoría. Estos autores proponen acercarse al conocimiento científico, fortalecer las destrezas, actitudes y aptitudes pedagógicas, así como trazar planes y políticas para la formación y la creación de un sistema de créditos para su cualificación en y desde las IEs. Pero un poco más allá, se puede localizar la propuesta de Margalef (2005), quien considera que la FPPU se debe orientar hacia las funciones sociales del conocimiento, la mejora de pro- cesos, la acreditación de universidades, el desarrollo de la identidad del profesor universitario y la redefinición de la enseñanza, estableciendo una reflexión y relación constante entre teoría, práctica y realidad cotidiana. Y en esta dirección, Maquilón Sánchez y García Sanz (2010) y Baute Álvarez e Iglesias León (2011) señalan que se requiere formación con la perspectiva hacia la función social y las competencias generales del profesor, en contenidos curriculares y de contexto; se debe preparar para: trabajar en equipo con diversos profesionales, realizar tutorías, planificar la investigación, aplicar metodologías innovadoras, utilizar las TIC, desarrollar y evaluar proyectos de investigación, dominar el inglés, practicar y promover ejercicios democráticos, así como para realizar la sistematización de sus experiencias de formación.

Un poco más centrados en la profesionalización del docente, González Tirados y González Maura (2007) concluyen en sus trabajos de investigación que el profesor universitario requiere conocimientos, habilidades y valores para ser eficiente, ético y responsable; sugieren los diagnósticos como punto de partida para las estrategias y programas de formación mediante diferentes alternativas flexibles y contextualizadas. Otra investigación desarrollada por Torra Bitlloch et al. (2013) encuentra que las particularidades y coincidencias de sus respectivos contextos universitarios permiten diseñar la oferta de formación permanente en las competencias comunicativa, metodológica, interpersonal, de planificación y gestión de la docencia, de trabajo en equipo e innovación y formación para la evaluación personal e institucional.

Zabalza Beraza (2013), por su parte, señala que se deben crear sistemas coherentes de formación permanente capaces de responder a las demandas que se les plantean en diversos ámbitos, buscando atraer, seleccionar, desarrollar, formar, incentivar y retener buenos profesores. Esta condición es posible siempre que no se oriente una identidad institucional desde principios éticos, conocimiento disciplinar, funciones, expectativas y competencias pedagógicas requeridas para su desempeño.

Finalmente, Abril, Ariza, Quesada y García (2014) plantean que la oferta para la FPPU se debe centrar en habilidades didácticas. Para ello, las IEs pueden realizar foros con expertos, favorecer el uso de materiales de apoyo y prácticas de enseñanza con otros docentes. 
Estos autores concluyen que programas formativos con adecuadas competencias, estrategias y recursos didácticos facilitan la interdisciplinariedad, el aprendizaje colaborativo y el clima en el aula.

Producto de las acciones reflexivas, analíticas y críticas que se han realizado a los estudios anteriores y a modo de síntesis analítica, se extraen las líneas gruesas que describen el estado de la discusión y desarrollo temático actual de la formación permanente del profesorado universitario. Asimismo, se resaltan las rutas metodológicas utilizadas en y para estudios de la temática (tabla 2).

Tabla 2.

Síntesis analítica del estado de la discusión y desarrollo temático actual de la FPPU

\begin{tabular}{|c|c|c|}
\hline & $\begin{array}{l}\frac{n}{0} \\
\frac{0}{0} \\
\frac{0}{0} \\
0 \\
0\end{array}$ & $\begin{array}{l}\text { Pensamiento espontáneo del profesorado } \\
\text { Rupturas y conflictos en departamentos encargados de la formación } \\
\text { Ausencia de tiempos para la formación } \\
\text { Ausencia de uso de Tic } \\
\text { Formación del profesorado, de los responsables administrativos que la financian } \\
\text { y de los académicos que planifican la formación permanente }\end{array}$ \\
\hline 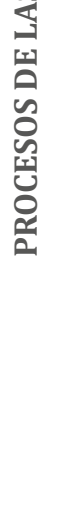 & 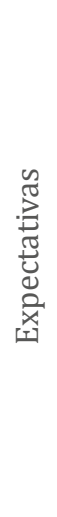 & $\begin{array}{l}\text { Fomentar un perfil profesional esperado según el contexto universitario } \\
\text { Desarrollar la identidad de la profesión en el profesorado } \\
\text { Crear departamentos de formación desde las exigencias de los sistemas de evaluación } \\
\text { Crear programas de impacto científico, pedagógico y social } \\
\text { Reconocer la percepción social del profesor en su responsabilidad } \\
\text { social y su rol en la docencia y la investigación } \\
\text { Responder a necesidades, retos de la sociedad actual y uso de recursos técnicos y tecnológicos } \\
\text { Trabajar con los agentes del proceso de desarrollo profesional } \\
\text { Desarrollar la competencia curricular del profesorado (liderazgo, gestión administrativa, planificación, } \\
\text { relaciones interpersonales, entre otros conocimientos, habilidades, valores y actitudes) }\end{array}$ \\
\hline 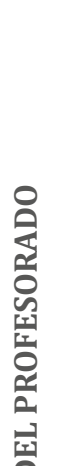 & 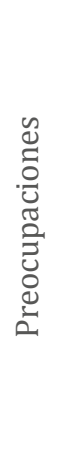 & $\begin{array}{l}\text { Innovación en la enseñanza } \\
\text { Dominio y comprensión del proceso de enseñanza-aprendizaje en contexto } \\
\text { Uso de las Tic en la gestión del aula y la relación con el proceso enseñanza-aprendizaje-evaluación } \\
\text { Capacidad de fusionar teoría, práctica e investigación } \\
\text { Atender a los cambios socioeconómicos, culturales, políticos, } \\
\text { tecnológicos y científicos de la sociedad globalizada } \\
\text { Uso de metodologías didácticas que desarrollen las capacidades del alumnado } \\
\text { Dominio metodológico de los procesos de investigación } \\
\text { Poder atender las expectativas de estudiantes, directivos y pares académicos }\end{array}$ \\
\hline 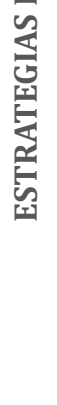 & 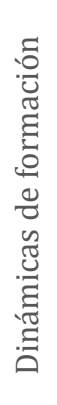 & $\begin{array}{l}\text { Ofertas contextualizadas desde las propias IES o el sector } \\
\text { Directrices teórico-prácticas para su rol en asesorías } \\
\text { Espacios para la reflexión acerca de la teoría, la práctica y la realidad cotidiana } \\
\text { Formación en la función social, cultural y competencias generales } \\
\text { Conocimientos, habilidades y valores para ser eficiente, ético y responsable } \\
\text { Formación en competencia comunicativa, metodológica, interpersonal, planificación y gestión de la } \\
\text { docencia, trabajo en equipo e innovación y formación para la evaluación personal e institucional. } \\
\text { Sistemas coherentes en la formación permanente }\end{array}$ \\
\hline
\end{tabular}


Los estudios revisados presentaron variadas rutas metodológicas, como se muestra en la tabla 3.

Tabla 3.

Rutas metodológicas de investigaciones sobre la FPPU

\begin{tabular}{llll}
\hline \multicolumn{1}{c}{ CUALITATIVAS } & \multicolumn{1}{c}{ CUANTITATIVAS } & \multicolumn{1}{c}{ TÉCNICAS } & \multicolumn{1}{c}{ POBLACIÓN } \\
\hline Investigación guiada & Estudios experimentales & Encuesta & Estudiantes de \\
Fenomenología & Estudios de caso & Observación & formación inicial \\
Análisis de discurso & & Entrevista & Profesores universitarios \\
Narrativas & & Grupos focales & Directivos de universidades, \\
Investigación-acción & & Relatos de experiencias & de departamentos \\
Estudios de caso & & académicos y estatales \\
\hline
\end{tabular}

Fuente: elaboración propia.

Líneas base para la formación

permanente del profesor universitario:

una discusión en proyectiva

Siguiendo la intencionalidad de aportar a la reflexión y el hallazgo de posibles rutas para repensar los procesos de formación permanente que demanda la universidad contemporánea, y específicamente la formación de los profesores del campo de la edu- cación física, emergen algunos lineamientos para el mejoramiento de estos procesos. Estos aspectos se subrayan como prioridades y elementos ineludibles en una estructura, proceso o programa de FPPU que se podrían desarrollar en y para todo campo de conocimiento. Se resaltan, entre otros, los ámbitos pedagógico, didáctico, psicopedagógico, curricular, administrativo e investigativo (tabla 4).

Tabla 4 .

Líneas base para estructurar un programa de FPPU

\begin{tabular}{cccccc}
\hline \multicolumn{5}{c}{ Líneas base para estructurar un programa de FPPU } \\
\hline $\begin{array}{c}\text { Ámbito } \\
\text { pedagógico }\end{array}$ & $\begin{array}{c}\text { Ámbito } \\
\text { didáctico }\end{array}$ & $\begin{array}{c}\text { Ámbito } \\
\text { psicopedagógico }\end{array}$ & $\begin{array}{c}\text { Ámbito } \\
\text { curricular }\end{array}$ & $\begin{array}{c}\text { Ámbito } \\
\text { administrativo }\end{array}$ & $\begin{array}{c}\text { Ámbito } \\
\text { investigativo }\end{array}$ \\
\hline
\end{tabular}

Fuente: elaboración propia.

Ámbito pedagógico. Estos aspectos se enfocan en la necesidad de atender y dinamizar procesos formativos poniendo en su centro el aprendizaje problémico; el desarrollo de competencias pedagógicas, didácticas, curriculares, comunicativas, científicas, sociales, afectivas y conversacionales; manteniendo el sentido de la formación contemporánea desde una actualidad cultural, social, científica, humanista, estética, ética y política que asiste la realidad y el contexto de cada universidad.

Ámbito didáctico. Han de buscar medir el impacto social, científico, pedagógico, uso de las TIC en las actuaciones académicas de los profesores y el aprender a combinar prácticas o asesorías en docencia e investigación. Desarrollar habilidades para generar motivación, inclusión, equidad, participación y reconocimiento del desempeño académico de sus estudiantes; promover diferentes ambientes de aprendizaje, la solución de conflictos y el favorecimiento del contacto con la vida cotidiana en el aula, la empresa y la sociedad, mediante estrategias de trabajo cooperativo, interdisciplinario, en equipo y por subgrupos, fusionando teoría, práctica e investigación. 
Ámbito psicopedagógico. Es de relevancia realizar diagnósticos para identificar el perfil emocional de profesores y estudiantes. Favorecer acciones para que los profesores desarrollen habilidades en creatividad, innovación y gestión del aula y, así, trascender estructuras tradicionales del proceso de enseñanza-aprendizaje, descubriendo nuevas formas de despertar interés en el saber enseñado, usando técnicas para la inclusión, la equidad y la toma de conciencia sobre su responsabilidad social en contextos nuevos y retadores.

Ámbito curricular. Los programas de FPPU deben estar estructurados por competencias cognitivas, metacognitivas, comunicativas, gerenciales, sociales, afectivas, pedagógicas, didácticas, científicas, uso de TIC, lectoescriturales y el desarrollo de habilidades para preparar, presentar y socializar su conocimiento. Los planes curriculares de formación profesional ofrecidos deben ser flexibles, dinámicos, participativos, concertados, incluyentes, transdisciplinares y con formación en una segunda lengua. Además, es importante que los profesores tengan acceso a materiales virtuales, uso de bases de datos y laboratorios, trabajo en equipo con investigadores externos o internos en IES. De esta manera se fomentan relaciones interpersonales y se abre la posibilidad de establecer juicios críticos frente a su proceso de formación.

Ámbito administrativo. Las investigaciones consultadas y referenciadas proponen una ruta centrada en departamentos encargados de la FPPU, y otra, en las acciones empleadas para tal fin. Estos departamentos deben contar con una estructura organizacional, liderazgo, flujo de información y comunicación, donde el profesor pueda encontrar apoyo, acompañamiento y motivación para su desempeño. Como acciones, deben evaluar sus propias actividades pedagógicas y académicas con respecto a la motivación que tiene el profesorado para la visibilidad de su ejercicio profesional y consolidar el perfil de producción académica de cada integrante.

Ámbito investigativo. Los estudios analizados plantean que la formación permanente debe capacitar al profesor para la innovación, visibilización de sus investigaciones, formación ciudadana, responsabilidad social, dominio de un segundo idioma, uso de TIC, gestión de sus publicaciones, desarrollo de habilidades para el liderazgo científico, trabajo en equipo e interdisciplinario, trabajo cooperativo con diferentes investigadores y universidades, uso de laboratorios, habilidades para comunicarse y visitar empresas, entre otras intenciones. Estos elementos deben permear y potenciar la producción, divulgación y pertinencia del nuevo conocimiento; lo mismo que la creación de semilleros, líneas y grupos de investigación específicos en la formación inicial, permanente y continuada del profesor universitario. Estas, a la vez, son algunas preocupaciones de las IEs para afrontar, emprender o desarrollar la FPPU.

En tal sentido, la puesta en marcha de estos lineamientos implica para las IEs y los responsables de la toma de decisiones la formulación de políticas que permitan conformar grupos de administradores que promuevan el flujo de información y comunicación de la oferta de formación de manera permanente, amplia y variada en temáticas a partir de diagnósticos reales de la demanda; con actividades que cuenten con materiales, talento humano y recurso financiero para llevarlas a cabo, de tal forma que se favorezcan el bienestar, la satisfacción, la motivación y el reconocimiento por ser profesor universitario.

Es válido entonces continuar la indagación en contexto por la reflexión pedagógica que hacen profesores noveles y expertos de cara a su rol profesional, pues como lo plantea Salazar Díaz (2015) las interacciones pedagógicas (en estas ubicamos las didácticas, psicopedagógicas y curriculares, entre otras) obedecen en gran medida a actuaciones horizontales y bilaterales en comunidades académicas, reconociendo las posibilidades de comunicación e interacción simétrica o asimétrica, todo esto, como escenario donde sea posible identificar su dominio desde la comprensión, transformación, instrucción, evaluación, reflexión y nueva comprensión de sus acciones, identificando el conocimiento de contenido que posee, el del saber pedagógico general, el curricular, el educativo, de sus estudiantes y del contexto.

Estos planteamientos también han sido señalados por investigadores como Palomero Pescador (2002), cuando comparte la trazabilidad de la revista interuniversitaria de formación del profesorado, evidenciando acciones como congresos y seminarios en universidades para ocuparse de cualificar el desempeño del profesor en este nivel educativo. En otro trabajo 
del mismo autor (2003) se resaltan los cambios que se han producido en su contexto desde la modalidad y componentes de la formación permanente, producto de la implementación de sistemas de evaluación del desempeño de los profesores en las universidades, definiendo con fuerza la formación psicopedagógica para que atiendan su misión de formar profesionales competentes y ciudadanos libres, ilustrados, respetuosos, críticos y tolerantes.

Marín Sánchez y Teruel Melero (2004) invitan a tener presente en la FPPU la necesidad de responder a demandas sociales del aprendizaje universitario y atender la dicotomía docencia-investigación, lo que sustenta su necesidad. En esta vía, Palomero Fernández (2009) puntualiza en formar al profesorado en competencias sociales y emocionales, resaltando actitudes como disposición fenomenológica, autonomía, responsabilidad, independencia de criterio y disposición cooperadora, con el fin de monitorear el impacto social que una propuesta formativa genera en el contexto intervenido.

El recorrido realizado plantea reflexiones ineludibles para la creación de un programa de FPPU, genera además motivaciones para poner en prospectiva rutas o líneas de investigación que potencien esta intencionalidad en pro tanto de los distintos actores de la comunidad, la institución educativa misma, como los proyectos de ciudad, país y región en los contextos en que se inscriben las universidades. Así se le apuesta a la responsabilidad social universitaria como fundamento misional central de toda universidad. Igualmente se hace el llamado a las IEs a darle la relevancia que la FPPU merece, dado que es aquí, a este ejercicio, a esta proyectiva, a estas dinámicas, en fin, a estas realidades, a quienes se les asigna la responsabilidad de la calidad de la educación en instancias superiores.

Para dinamizar dicha proyectiva se requiere como factor determinante escuchar la voz del profesorado, entender y atender sus preocupaciones, necesidades, intereses, gustos y posibilidades, además del reconocimiento de tiempos y espacios en los planes laborales. Se debe permitir la articulación a procesos de trabajo cooperativo interno o en redes externas para el intercambio de conocimiento local e internacional.

Los postulados planteados en los diferentes estudios que abonan esta discusión, tienen resonancia con apreciaciones de Zabalza (2009) al referirse a la necesidad de formar al profesorado universitario desde competencias generales y específicas, que le permitan la construcción de su verdadera identidad desde un compromiso personal y una responsabilidad social e institucional. Es así como se hace imprescindible reconocer que los fundamentos anteriores no pueden estar alejados de la necesidad de que el profesor se forme en condiciones, características y requerimientos de su disciplina, informe y proyecte objetivos, conozca e innove métodos, genere alternativas de investigación y de reflexión sobre su propia práctica, entre otros aspectos y dinámicas específicas de su campo.

Cabe mencionar, finalmente, una estrategia y experiencia puntual para la formación del profesorado que es expuesta por Bozu e Imbernon Muñoz (2016) como es el posgrado en iniciación a la docencia universitaria, desarrollado con profesores principiantes de la Universidad de Barcelona, para que los profesores noveles adquieran hábitos, recursos y herramientas ante la mejora continua y la innovación en su práctica docente. Resaltan otras estrategias como programas teórico-prácticos de iniciación, procesos de mentoría con profesores expertos, y concluyen que el profesor universitario necesita de "una formación pedagógica general y sobre todo de una formación didáctico-metodológica básica que les permita ejercer la función docente. No basta solo con tener una formación científica, de especialidad y con dominar los conocimientos propios de esa área curricular" (p. 487) y dejan en evidencia que ejercer el rol de profesor universitario con calidad en la enseñanza requiere una sólida formación psicopedagógica.

\section{CONCLUSIONES}

Los estudios analizados permitieron identificar contenidos y metodologías desarrolladas por instituciones de educación superior para cualificar el rol profesional de su profesorado que develan rutas pedagógicas, didácticas, psicopedagógicas, curriculares, administrativas e investigativas asumidas en las universidades para la FPPU. En ellas se percibe una estructuración desde planteamientos institucionales que, pareciera, desconocen su episteme, la voz de los profesores universitarios y los contextos de realidad donde se escenifica la enseñanza universitaria. 
Quizás esta situación posibilite asumir otras rutas desde la óptica del profesor universitario - puesto que pareciera se le desconoce como actor protagónico-, y ello permita un diálogo y consenso abierto entre las IES y sus profesores, para contribuir a la educación como acontecimiento ético y compromiso de responsabilidad social universitaria según el contexto donde se imparta, y pueda así contar con nuevas miradas, enfoques y perspectivas en la cualificación del docente universitario.

Por lo tanto, los resultados del análisis de las investigaciones consultadas destacan la imperiosa necesidad de crear y ofrecer a los profesores políticas institucionales, recursos, reconocimientos, programas de formación innovadores en docencia, investigación, proyección social, formación ciudadana y responsabilidad social universitaria. Del mismo modo, sugieren asignar tiempos para la creatividad en la enseñanza, espacios para la innovación curricular, opciones de flexibilidad curricular, tiempos y espacios para la reflexión cultural, académica, pedagógica, científica, posibilidades para observación de prácticas exitosas y de sus propias prácticas de enseñanza.

Para ello, se deberán diseñar estrategias de reconocimiento de los tiempos requeridos para la formación permanente o posgradual, seguimiento del impacto generado a partir de la implementación de las citadas rutas teóricas y prácticas; y en especial, que cada universidad diseñe su propio plan de formación permanente y posgradual del profesorado, entendiendo las lógicas de cada campo de saber y área de formación profesional.

A la vez, desde las investigaciones consultadas, emergen estrategias de formación que sugieren la realización de foros, conferencias de expertos, talleres, proyectos de trabajo, seminarios, congresos o técnicas en subgrupos, seminarios permanentes, según sea el perfil de profesor en cada universidad, y contar con un programa institucional que comprenda la inducción, la iniciación y el desarrollo del rol docente. Y se puede precisar que la tarea asignada a los departamentos encargados de la FPPU consiste en realizar diagnósticos internos que permitan identificar necesidades de formación y articular sus demandas, hacia la búsqueda de cualificación que atienda las necesidades institucionales frente al desempeño de los profesores.

\section{REFERENCIAS}

Abril, A. M., Ariza, M. R., Quesada, A. y García, F. J. (2014). Creencias del profesorado en ejercicio y en formación sobre el aprendizaje por investigación. Revista Eureka Sobre Enseñanza y Divulgación de las Ciencias, 11(1), 22-33.

Baute Álvarez, L. M., e Iglesias León, M. (2011). Sistematización de una experiencia pedagógica: la formación del profesor universitario. Revista Pedagogía Universitaria, 16(1), 36-49. Recuperado de http://cvi.mes. edu.cu/peduniv/index.php/peduniv/article/view/60.

Belando Montoro, M. R. (1999). Los profesores del siglo xxI y la calidad de la enseñanza universitaria: en torno a la formación. Revista Electrónica Interuniversitaria de Formación del Profesorado, 2(1), 1-8.

Biscarri Gassio, J., Filella Guiu, G. y Jové Monclús, G. (2006). Factores relacionados con la percepción de la calidad docente del profesorado universitario. Revista Interuniversitaria de Formación del Profesorado, 20(3), 287-309.

Bozu, Z. (2009). El profesorado universitario novel y su proceso de inducción profesional. Magis. Revista Internacional de Investigación en Educación, 1(2), 317-328.

Bozu, Z. e Imbernon Muñoz, F. (2016). La formación docente en momentos de cambios: ¿Qué nos dicen los profesores principiantes universitarios? Revista de Curriculum y Formación del Profesorado, 20(3), 467-492. Recuperado http://diposit.ub.edu/dspace/ handle/2445/114965

Bozu, Z. y Canto Herrera, P. J. (2009). El profesorado universitario en la sociedad del conocimiento: competencias profesionales docentes. Revista de Formación e Innovación Educativa Universitaria, 2(2), 87-97. Recuperado de http://tecnologiaedu.us.es/mec2011/htm/ mas/3/31/26.pdf.

Caballero Rodríguez, K. (2013). La formación del profesorado universitario y su influencia en el desarrollo de la actividad profesional. REDU: Revista de Docencia Universitaria, 11(2), 391-412.

Cano González, R. y Revuelta Guerrero, C. (1999). La formación permanente del profesorado universitario. Revista Electrónica Interuniversitaria de Formación del Profesorado, 2(1), 56-62. Recuperado de http://dialnet.unirioja.es/servlet/ culo? codigo $=2794992 \&$ orden $=181862 \&$ info=link.

Carrasco, J. B. y Calderero Hernández, J. F. (2000). Aprendo a investigar en educación. Madrid: Anzos. 
Coharik Chamlian, H. (2003). Docência na universidade: professores inovadores na USP. Cadernos de Pesquisa, 118(1), 41-64. https://doi.org/10.1590/ S0100-15742003000100003.

Concepción Cuétara, P. M., Fernández Cruz, M. y González González, D. (2014). La construcción de un cuestionario para la detección de necesidades formativas del profesorado novel. Pedagogía Universitaria, 19(1), 1-29.

Escudero Muñoz, J. M. (1999). La formacion permanente del profesorado universitario. Revista Interuniversitaria de Formación del Profesorado, 34(1), 133-157.

Ferreres Pavía, V. (2001). El desarrollo profesional del profesorado universitario: Circunstancias, problemas y propuestas. Profesorado, Revista de Currículum y Formación del Profesorado, 5(2), 1-26. Recuperado dehttp://www.opech.cl/bibliografico/Doc_Docente/ El_desarrollo_profesional_del_profesorado_universitario_circunstancias_problemas_y_propuestas.pdf.

Fraile Aranda, A. (2004). Un cambio democrático en las aulas universitarias: una experiencia en la formación del profesorado de educación física. Contextos Educativos, 7(6-7), 213-234.

Fraile Aranda, A. (2010). Construyendo un camino que ayude a conocer la importancia de la autonomía en la formación del profesorado. Revista Española de Educación Física y Deportes, 14(5), 9-23.

García Nieto, N. (2008). La función tutorial de la universidad en el actual contexto de la Educación Superior. Revista Interuniversitaria de Formación del Profesorado, 22(1), 21-48.

García Ruso, H. M. (1997). La formación del profesorado de educación física: problemas y expectativas. Barcelona: Editorial INDE.

Gil Pérez, D., Beléndez Vásquez, A., Martín García, A. y Martínez Torregrosa, J. (1991). La formación del profesorado universitario de materias cientificas: contra algunas ideas y comportamientos de sentido común. Revista Interuniversitaria de Formación del Profesorado, 12(2), 43-48.

Giner Cotino, F. J. y Giner Cotino, V. (1998). La formación permanente del profesorado a través del asesoramiento pedagógico en un centro de profesores. Revista Interuniversitaria de Formación del Profesorado, 31(1), 161-171. Recuperado de http://dialnet.unirioja.es/ descarga/articulo/117975.pdf.

González Tirados, R. M. y González Maura, V. (2007). Diagnóstico de necesidades y estrategias de formación docente en las universidades. Revista Iberoamericana de Educación, 43, 1-14. Recuperado dehttp://dialnet. unirioja.es/servlet/articulo?codigo $=2358650$.

Hadi, E. y Cabrera, F. (1999). El perfeccionamiento del profesorado en Chile. Revista Interuniversitaria de Formación del Profesorado, 35, 95-101. Recuperado de http:// dialnet.unirioja.es/descarga/articulo/118036.pdf.

Imbernon, F. (1989). La formación inicial y la formación permanente del profesorado: dos etapas de un mismo proceso. Revista Interuniversitaria de Formación del Profesorado, 6(4), 487-499. Recuperado dehttp://dialnet.unirioja.es/servlet/articulo?codigo $=117680$

Imbernon, F. (1999). Responsabilidad social. Revista Interuniversitaria de Formación del Profesorado, 34, 123-132.

López Rodríguez, N. M. (2012). Una mirada al desarrollo docente y la formación del profesorado en la Universidad Santo Tomás seccional Bucaramanga. Espiral, Revista de Docencia e Investigación, 2(2), 37-56. Recuperado dehttp://revistas.ustabuca.edu.co/index.php/ ESPIRAL/article/view/145.

Madrid Izquierdo, J. M. (2005). La formación y la evaluación docente del profesorado universitario ante el espacio europeo de educación superior . Educatio, 23, 49-68.

Maquilón Sánchez, J. J. y García Sanz, M. P. (2010). El futuro de la formación del profesorado universitario. Revista Electrónica Interuniversitaria de Formación del Profesorado, 14, 17-26. Recuperado de http://www.aufop. com/aufop/home/.

Margalef García, L. (2005). El reto de la formación del profesorado universitario: ¿hacia dónde vamos? ¿convergencia o desconvergencia? Reifop. Revista Electrónica Internuniversitaria de Formación del Profesorado, 8(6), 53-57.

Margalef García, L. y Álvarez Méndez, J. M. (2005). La formación del profesorado universitario para la innovación en el marco de la integración del espacio europeo de educación superior. Revista de Educación, 2(337), 51-70.

Marín Sánchez, M. y Teruel Melero, M. P. (2004). La formación del docente universitario: necesidades y demandas desde su alumnado. Rifop: Revista Interuniversitaria de Formación del Profesorado, 18(2), 137-151.

Mayor Ruiz, C. (1997). El perfeccionamiento de los profesores universitarios principiantes a juicio de sus alumnos. Revista Interuniversitaria de Formación del Profesorado, 30(4), 127-149.

Mayor Ruiz, C. (2003). Dinámicas formativas para la docencia universitaria. En Enseñanza y aprendizaje en la educación superior (pp. 181-209). Barcelona: Octaedro. 
Medina Moya, J. L., Jarauta Borrasca, B. y Urquizu Sánchez, C. (2005). Evaluación del impacto de la formación del profesorado universitario novel: un estudio cualitativo. Revista de Investigación Educativa, 23(1), 205-238.

Mérida Serrano, R. (2013). La controvertida aplicación de las competencias en la formación docente universitaria. Revista de Docencia Universitaria, 11(1), 185-212.

Padierna Cardona, J. C. (2010). El perfil pedagógico competente: un análisis a la luz del formador de formadores en la Licenciatura en Educación Física y Deportes de la Universidad San Buenaventura. Educación Física y Deporte, 29(2), 269-276.

Palomero Fernández, P. (2009). Desarrollo de la competencia social y emocional del profesorado: una aproximación desde la psicología humanista. Reifop. Revista Electrónica Interuniversitaria de Formación del Profesorado, 12(2), 145-153.

Palomero Pescador, J. E. (2002). Indicadores de calidad, resultados y línea editorial. Quince años de historia de la revista interuniversitaria de formación del profesorado. Revista Interuniversitaria de Formación del Profesorado, 44(2), 223-266.

Palomero Pescador, J. E. (2003). La formación psicopedagógica del profesorado universitario en la España de hoy: Tiempos de cambio. El Guiniguada, 12(1), 105-120.

Pérez Miranda, R. y Gallego Badillo, R. (2006). Concepciones sobre pedagogía y didáctica de un grupo de docentes . Informe de investigación. Educación y Pedagogía, 18(44), 127-140.

Piña Loyola, C., Pez Camerón, P., León Rodríguez, A., Leyva Gainza, Y., González Pérez, F. y León Sánchez, Y. (2014). La formación del profesor universitario: un profesional en superación constante. Medisur, 12(1), 241-248.

Rodríguez Izquierdo, R. M. (2003). Reaprender a enseñar: una experiencia de formación para la mejora continua de la docencia universitaria. Revista Interuniversitaria de Formación del Profesorado, 17(2), 79-94.

Rué, J. (2013). Formación docente del profesorado universitario. Una perspectiva internacional. REDU: Revista de Docencia Universitaria, 11(3), 17-22.

Ruiz Corbella, M. (1999). La educación para la ciudadanía europea en la formación del profesorado, una propuesta. Revista Interuniversitaria de Formación del Profesorado, 35(1), 103-114. Recuperado de http:// dialnet.unirioja.es/descarga/articulo/118037.pdf.
Salazar Díaz, C. A. (2015). Razonamiento pedagógico de docentes de Licenciatura en Cultura Física, Recreación y Deporte en investigación formativa. Lúdica Pedagógica, 22(2), 111-121.

Sánchez, E. F. y Flores, J. Y. (2013). La formación docente y el desarrollo curricular como alternativas de innovación educativa. Revista Educare, 17(1), 166-181.

Solá Fernández, M. (2004). La formación del profesorado en el contexto del espacio europeo de educación superior. Avances alternativos. Revista Interuniversitaria de Formación del Profesorado, 18(3), 91-105.

Tomé, M. A. de la Cruz. (2000). Formación pedagógica inicial y permanente del profesor universitario en España: reflexiones y propuestas. Revista Interuniversitaria de Formación del Profesorado, 38(4), 19-35. Recuperado dehttp://www.aufop.com/aufop/uploaded_files/articulos/1223489926.pdf.

Torra Bitlloch, I., Márquez Cebrián, M. D., Páges Costas, T., I Ysuar, P. S., García Campos, R., Molina Luque, F., ... Sangrá Morer, A. (2013). Retos institucionales de la formación del profesorado universitario. REDU: Revista de Docencia Universitaria, 11(1), 285-309.

Vaquero Barba, Á. (2000). Claves para la formación del profesorado de educación física desde una perspectiva crítica: análisis de un proceso formativo y alternativas. Bilbao: Universidad del País Vasco.

Yániz Álvarez, C. (2008). Las competencias en el currículo universitario. Revista de Docencia Universitaria, 4, 14. Recuperado de http://www.um.es/ead/Red_U/m1/ yaniz.pdf.

Zabalza, M. Á. (2003). Innovación en la enseñanza universitaria. Contextos Educativos: Revista de Educación, 6-7(1), 113-136. Recuperado de http://dialnet.unirioja.es/descarga/articulo/1049473.pdf.

Zabalza, M. Á. (2009). Ser profesor universitario hoy. $L a$ Cuestión Universitaria, 5(5), 69-81. Recuperado de http://unmotivo.com/lcu/grafica/articulos/imgs_ boletin_5/pdfs/LCu5-7.pdf.

Zabalza Beraza, M. (2000). El papel de los departamentos universitarios en la mejora de la calidad de la docencia. Revista Interuniversitaria de Formación del Profesorado, 38, 47-66.

Zabalza Beraza, M. (2013). La formación del profesorado universitario. REDU: Revista de Docencia Universitaria, 11(3), 11-15. 